\title{
Projecto Angolano de Formação e Desenvolvimento da Dermatologia: Avanços e Desafios
}

\author{
Lídia P. de Almeida Voumard1,2 \\ 'Ministério da Saúde, Angola \\ ${ }^{2}$ Faculdade de Medicina da Universidade Agostinho Neto, Luanda, Angola
}

RESUMO - Introdução: Apesar do fardo e das repercussões físicas, psicológicas e sociais que as doenças da pele representam, o desenvolvimento da Dermatologia e a formação de técnicos nessa disciplina defrontam em Angola sérios desafios, com repercussões no acesso e na qualidade dos serviços e cuidados prestados a população. Metodologia: Foi realizado um estudo retrospectivo do Projecto de Formação e Desenvolvimento da Dermatologia, iniciado em 2010 com o apoio do Ministério da Saúde e do Colégio Angolano de Dermatologia e Venereologia (CADV). O projecto tem por objectivo a formação de mais dermatologistas e de aumentar o acesso dos doentes a Serviços de referência. São apresentados dados sobre a formação dos internos, bem como outros ganhos e limitações do Projecto. Resultados: Até 2013, o internato médico complementar em Dermatologia estava reservado apenas a um Serviço de Dermatologia em Luanda, tendo formado oito dermatologistas desde 1975. Em 2013, cinco novos Serviços Regionais, abertos em Cabinda, Malange, Huambo, Benguela e Huíla, iniciaram a formação de especialistas, aumentando em 2017 a 26 o número total de internos em formação. Conclusão: No contexto de Angola, a descentralização dos serviços clínicos de formação, a afinação periódica do programa de pós-graduação e a promoção activa de atitudes e actividades de pesquisa são elementos chave no desenvolvimento da Dermatologia e na prestação de serviços de referência mais próximos das populações.

PALAVRAS-CHAVE - Angola; Dermatologia/educação.

\section{Angolan Project of Formation and Development of Dermatology: Advances and Challenges}

ABSTRACT - Introduction: Despite the burden of skin disorders and their physical, psychological and social repercussions, the development of Dermatology and the training of technicians in this discipline face serious challenges in Angola, with a negative impact on the access and quality of care and services provided to the population. Methods: A retrospective study of the Dermatology Training and Development Project was carried out. The Project, launched in 2010 with the support of the Ministry of Health and the Angolan College of Dermatology and Venereology (CADV), aims at increasing the number of dermatologists and the access of patients to clinical referral services. Data on the training activities, other gains and limitations of the Project are presented. Results: Up to 2013, the residency in Dermatology was confined to a single Service in Luanda, which trained eight dermatologists from 1975 to 2013. Five new Regional Dermatology services were opened in 2013 in Cabinda, Malange, Huambo, Benguela and Huila. As a result the total number of residents in Dermatology increased to 26 in 2017. Conclusion: In the context of Angola, the decentralization of clinical training services, the periodic update of the postgraduate training program and the active promotion of research attitudes and activities are key factors for the development of Dermatology and the provision of referral services closer to the populations.

KEYWORDS - Angola; Dermatology/education.

Correspondência: Lídia P. de Almeida Voumard

Faculdade de Medicina da Universidade Agostinho Neto

Av. Hoii ya Henda - Hospital Américo Boavida

Luanda - Angola

Tel.: +244935133219

E-mail: lidiavoumard2003@yahoo.fr

DOI: https://dx.doi.org/10.29021/spdv.76.3.957
Recebido/Received

30 Maio/May 2018

Aceite/Accepted

14 Junho/June 2018 


\section{Artigo Original}

\section{INTRODUÇÃO}

Tanto nos países desenvolvidos como nos países em desenvolvimento, as doenças da pele são frequentes e interferem de forma significativa na saúde física, psicológica e social dos indivíduos e das populações, sobretudo quando surgem atrasos ou erros de diagnóstico e tratamento. ${ }^{1}$ Em África, o peso dessas doenças contrasta com o baixo número de profissionais qualificados (um dermatologista para mais de 400000 habitantes em média) e a sua habitual concentração em só uma ou duas cidades. ${ }^{2}$

Em Angola, pelo menos 30 \% dos pacientes que acorrem aos postos e centros de saúde apresentam lesões da pele. As deficiências de higiene pessoal e a precariedade do saneamento do meio estão implicadas nas patologias infecciosas da pele, sendo igualmente comuns outras afecções como a acne, dermatites de causas diversas e distúrbios da pigmentação. ${ }^{3}$

Por outro lado, ocorrem também patologias mais complexas, de origem genética, imunológica, tumoral, e doenças tropicais negligenciadas (DTNs) como a hanseníase, a oncocercose, a filariose linfática, as febres hemorrágicas, ${ }^{4}$ que requerem uma atenção mais diferenciada por parte de profissionais treinados e qualificados.

A eliminação da hanseníase, das filarioses e a prestação de cuidados médicos integrados a grupos vulneráveis tais como os albinos, as pessoas vivendo com o VIH/SIDA, exigem uma coordenação actuante entre os programas de saúde pública e a Dermatologia. ${ }^{4,5}$

A experiência adquirida em vários países mostra que as patologias mais simples e frequentes podem ser tratadas ao nível primário, se existirem as devidas competências. ${ }^{5}$ Entretanto, observa-se que muitos médicos de família e enfermeiros de primeira linha carecem da capacitação necessária para gerir as doenças da pele. ${ }^{6}$ Existe também a nível da atenção primária a tendência de superdiagnosticar algumas afecções em detrimento de outras, e de não saber diferenciar as afecções graves das lesões vulgares. ${ }^{1}$

Além disso, erros ou atrasos de tratamento podem resultar da carência de diagnóstico histopatológico, influenciando de forma negativa a evolução do paciente. , $7,8^{-1}$

Por todas essas razões, pensar no desenvolvimento da Dermatologia em Angola implica aumentar a qualidade dos cuidados primários de saúde, bem como o acesso a serviços clínicos de referência, devidamente preparados e equipados. A semelhança de outros países, ${ }^{9-11}$ a evolução da disciplina e seus avanços tecnológicos conduziram os dermatologistas angolanos a uma reflexão minuciosa sobre a formação local de mais especialistas, capazes de prestar cuidados de qualidade, bem como de formar outros quadros na disciplina.

Desde 1975 a 2013, o desenvolvimento da Dermatologia e formação dos internos de especialidade foram confinados ao Serviço de Dermatologia do Hospital Américo Boavida (HAB) em Luanda, com grandes limitações de ingresso no internato médico complementar da especialidade.
Além disso, até o início da década de 2010, a cirurgia, a ginecologia e obstetrícia, a medicina interna e a pediatria, foram as áreas de preferência da maioria dos médicos recém-graduados.

Em 2003, devido a falta de vagas e candidatos, só havia sete internos de dermatologia em formação e até 2013, apenas oito dermatologistas foram formados, todos radicados na cidade capital, Luanda, por razões históricas, familiares e profissionais.

Com o fim do conflito interno e a chegada da Paz em 2002, muitas transformações sociais e económicas surgiram progressivamente no País. Foram abertos, a partir de 2009, cinco novos Centros Hospitalares Regionais Universitários, em Cabinda, Malange, Huambo, Benguela e Huíla. ${ }^{12}$

Em 2009, a disciplina da Dermatologia na Faculdade de Medicina da Universidade Agostinho Neto passou a ter mais autonomia, visibilidade e capacidade de desenvolvimento, com a criação do Departamento de Ensino e Investigação (DEI) de Dermatologia, assim como, no ano seguinte, do Colégio Angolano de Dermatologia e Venereologia (CADV). ${ }^{13}$

Neste novo contexto, o Projecto de Desenvolvimento da Dermatologia ${ }^{14}$ surge em 2010 com o objectivo de formar mais especialistas e técnicos, de melhorar a sua distribuição geográfica e de dinamizar a investigação sobre os aspectos clínicos, epidemiológicos e estructurantes da disciplina no País.

São apresentados os primeiros resultados deste Projecto, bem como algumas lições aprendidas, constrangimentos e perspectivas da disciplina no contexto de Angola.

\section{METODOLOGIA}

Trata-se duma análise retrospectiva do Projecto de Desenvolvimento e Formação em Dermatologia, ${ }^{14}$ com destaque nas actividades realizadas entre 2010 e 2017. O Projecto surge na sequência das observações e lições tiradas de um estudo comparativo sobre a formação pré e pós-graduada em Dermatologia na China e em Angola. ${ }^{15}$ Foi elaborado por dermatologistas nacionais e aprovado em 2010 pelo Ministério da Saúde (MINSA), que nomeou para a fase de implementação uma Coordenadora do internato médico complementar em Dermatologia.

O diagnóstico da situação baseou-se na experiência do Serviço de Dermatologia do HAB e nas informações colhidas durante visitas aos cinco novos Centros Hospitalares Universitários Regionais. Foram tomadas em consideração as Directivas do Ministério da Saúde sobre a formação e o internato médico complementar hospitalar, a gestão hospitalar e a idoneidade dos serviços de formação. ${ }^{16-20}$

\subsection{Objectivo e estratégia do Projecto}

O objectivo principal do Projecto é de formar e fixar Dermatologistas a nível dos hospitais regionais e provínciais, com vista a oferecer, com qualidade, serviços e cuidados médicos da pele mais pertos das populações. 


\section{Artigo Original}

Foram propostas as seguintes acções estratégicas para alcançar este objectivo:

a) Harmonizar a nível nacional o currículo de formação.

b) Atribuir aos Dermatologistas formados, a missão de capacitar os médicos de família e técnicos médios para diagnosticar e tratar as patologias cutâneas mais simples e mais comuns a nível municipal e comunal.

c) Valorizar as actividades de pesquisa no programa de formação,

d) Criar cinco Serviços de Dermatologia nos Centros Regionais Hospitalares acima referidos, enquadrados por tutores com capacidade de formar internos de especialidade com a qualidade oferecida no Hospital Américo Boavida $(\mathrm{HAB})$.

e) Oferecer a outros hospitais centrais de Luanda (Josina Machel -HJM, Neves Bendinha -HNB, Hospital Militar-HM e Hospital Geral-HG) a oportunidade de aderir ao Projecto.

f) Prestar uma atenção especial aos mecanismos de supervisão, monitoria e avaliação da formação.

d) Criar parcerias estratégicas com as Faculdades Regionais de Medicina, as respectivas Delegações provinciais do MINSA e outras instituições, com o objectivo de reforçar a qualidade do ensino pré-graduado da disciplina e a prestação dos serviços clínicos.

\section{RESULTADOS}

\subsection{Acções estruturantes do Projecto}

As principais acções estructurantes em prol do desenvolvimento da disciplina são apresentadas na Tabela 1 .

O diagnóstico da situação da disciplina foi discutido e adoptado por todos os intervenientes como ponto de partida do Projecto. A criação em 2009 do Departamento de Ensino e Investigação (DEI) de Dermatologia da Faculdade de Medicina da Universidade Agostinho Neto (FMUAN) reforçou de forma notável as sinergias entre a Faculdade e o Serviço de Dermatologia do HAB. O currículo pré-graduado foi melhor ajustado ao perfil epidemiológico local, e os alunos passaram a ter no HAB aulas práticas mais efectivas, incluindo trabalhos de grupo.
Um ano mais tarde, o reforço técnico do Serviço de Dermatologia do $\mathrm{HAB}$, com o apoio da cooperação médica cubana, permitiu ao mesmo de assumir o seu papel de serviço clínico de referência terciária e centro normativo - metodológico para a formação pré e pós-graduada em Dermatologia-Venereologia.

Sob o impulso do Projecto, esses ganhos levaram a criação em 2010 do Colégio Angolano de Dermatologia e Venereologia (CADV), com o objectivo de mobilizar todos os Dermatologistas do País em prol da valorização da disciplina, formação contínua, pesquisa e exercício controlado, de forma a atingir os mais elevados padrões, para o benefício da saúde da população angolana. ${ }^{13}$

O fórum metodológico alargado organizado em 2013, pela Coordenação do Projecto, congregou os dermatologistas do Colégio Angolano de Dermato-Venereologia (CADV), quadros superiores do Ministério da Saúde (MINSA), Directores pedagógicos dos hospitais envolvidos, Bastonário da Ordem dos Médicos, Representantes da Cooperação Médica Cubana e tutores cubanos, com o objectivo de discutir as modalidades de criação de cinco novos Serviços de Dermatologia em Cabinda, Malange, Huambo, Benguela e Huíla (Fig. 1). Foram

\section{5: Serviço de Dermatologia do Hospital Américo Boavida, Luanda}

\section{9-2011: 5 Novas Faculdades de Medicina - Cabinda, Malange, Huambo, Benguela e Huíla \\ 2013: 5 novos Serviços Regionais de Dermatologia}

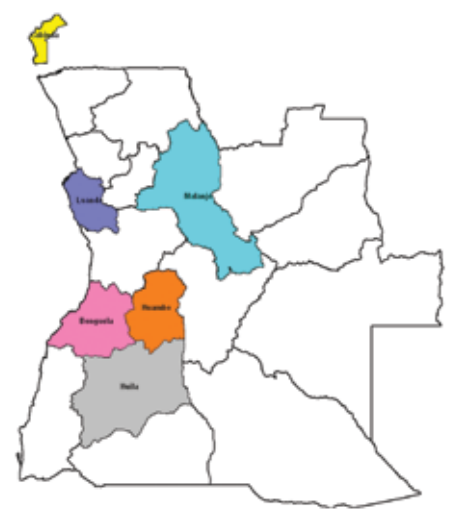

Figura 1 - Hospitais Central e Regionais oferecendo formação pré e pós-graduada em Dermatologia.

Tabela 1 - Principais acções em prol do desenvolvimento da disciplina.

PERÍODO

2009-2010

2009

2009

2010

2013

2014

2015

\section{ACTIVIDADES REALIZADAS}

Diagnóstico da situação da Dermatologia a nível nacional, elaboração e aprovação do Projecto.

- Reforço técnico do Serviço de Dermatologia do Hospital Américo Boavida (HAB).

- Criação do Departamento de Ensino e Investigação (DEI) de Dermatologia da FMUAN, em paralelo e sob impulso do Projecto.

- Criação do Colégio Angolano de Dermatologia e Venereologia (CADV).

- Fórum metodológico sobre os novos Serviços de Dermatologia e o currículo de formação dos internos de especialidade.

- Abertura dos cinco novos Serviços Regionais de Dermatologia.

- Início da formação dos primeiros internos a nível Regional, de acordo com a legislação e os regulamentos sobre a pós-graduação.

Apetrechamento dos Serviços de Dermatologia com novos equipamentos e materiais didácticos.

- Primeiro Simpósio Nacional de Dermatologia 


\section{Artigo Original}

propostos procedimentos e normas de organização e funcionamento desses serviços, bem como condições e mecanismos essenciais para uma abertura do internato médico complementar em Dermatologia. ${ }^{21}$

Em Outubro de 2013, na presença do Presidente do Conselho Nacional de Estudos Pós-graduados em Ciências Médicas (CNEPGCM), do Director Nacional dos Recursos Humanos do Ministério da Saúde, do Director Provincial da Saúde (DPS) e dos Directores dos Hospitais Regionais, foram abertos no Huambo pelo então Ministro da Saúde os cinco novos Serviços de Dermatologia dos Centros Regionais Hospitalares Universitários, com condições julgadas adequadas para realizar consultas ambulatórias e internamentos. ${ }^{22}$

Um ano depois foram instalados nesses Serviços novos meios de diagnósticos e terapêuticos, incluindo equipamentos de crioterapia, instrumentos de pequena cirurgia, dermatoscopia, acrescidos em Luanda de fototerapia e meios de alergologia e laboratório para exames directos. Os Serviços foram igualmente apetrechados com novos livros e manuais de referência, revistas, meios informáticos e fotográficos.

A ausência duma unidade central de histopatologia cutânea, prevista no Projecto, interfere contudo com a formação dos internos e a qualidade dos diagnósticos clínicos que necessitam de confirmação laboratorial (Fig. 2).

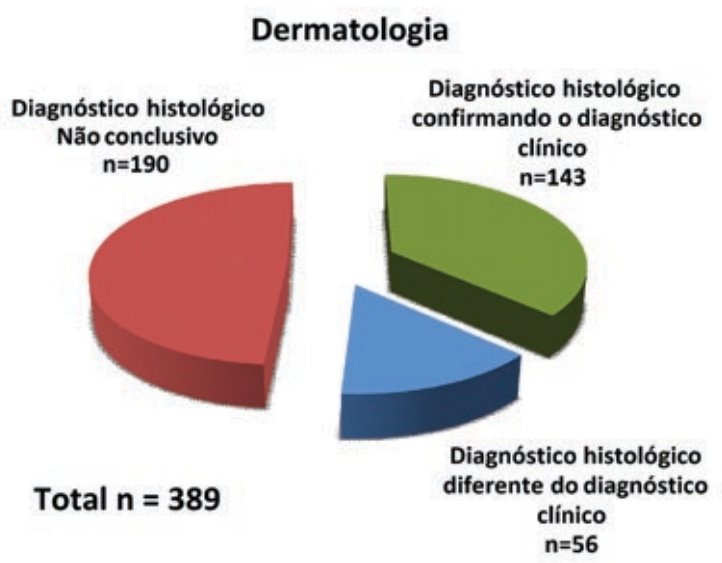

Figura 2 - Biópsias cutâneas no Serviço de Dermatologia do Hospital Américo Boavida (2016).

Os Serviços da Huíla e Huambo abriram consultas dedicadas ao seguimento dos albinos, casos de Hanseníase e dermatopediatria; no Huambo, na Huíla e em Benguela, os internos participaram em visitas multidisciplinares nos municípios, realizando actividades de informação, educação, comunicação (IEC) e assistência médica básica para doentes com patologia da pele.

Com adesão dos tutores e internos, a coordenação do projecto promoveu desde o início do Projecto uma colaboração activa com a Direcção Nacional dos Recursos Humanos, os Directores provinciais do MINSA, com a Ordem dos
Médicos de Angola (ORMED), a Cooperação Médica Cubana e as Faculdades Regionais de Medicina.

Com os novos Serviços Regionais a funcionar, os estudantes de medicina das novas Faculdades passaram a ver mais doentes com patologias cutâneas, levando a uma meIhoria qualitativa da formação pré-graduada na disciplina.

Foram criadas novas oportunidades de colaboração técnica para consolidar o desenvolvimento da disciplina, nomeadamente com Centros académicos em Portugal, Brasil, Moçambique, Tanzânia ${ }^{10}$ e China, ${ }^{15}$ bem como através da Liga Internacional das Sociedades de Dermatologia (ILDS) e Sociedade Internacional de Dermatologia (IDS).

\subsection{Formação dos internos de especialidade}

Para facilitar a aprendizagem e o seguimento dos internos, foi introduzido em 2013 um currículo de formação modular, dando especial atenção a acquisição de conhecimentos e competências práticas em áreas prioritárias, como a dermatocirurgia, dermatopatologia, crioterapia, micologia, fototerapia, dermatoscopia, oncologia e alergologia cutânea.

Durante o terceiro ano da formação, que conta quatro anos, foi formalizado um período de rotação de oito meses, com estágios em Serviços laboratoriais e clínicos oferecendo valências e formação não disponíveis no Serviço de Dermatologia do HAB. Foi igualmente acrescentado um estágio final adicional de seis meses fora do País, para acquisição de novas competências numa subespecialidade. ${ }^{21}$

Aproveitou-se a contratação dos dermatologistas cubanos responsáveis do ensino pré-graduado, para chefiar os novos Serviços clínicos, sinergia determinante para oferecer localmente a formação pós-graduada em Dermatologia.

Foram reforçadas as actividades de monitoria e avaliação: avaliação contínua dos internos pelos tutores, registo das actividades individuais pelos internos, visitas trimestrais de supervisão aos Centros de Formação pelos supervisores nacionais, avaliações teóricas/práticas contínuas, trimestrais e anuais sobre as matérias ensinadas, estágios bianuais de dois meses no HAB para os internos regionais, e exames anuais e finais com apresentação de currículo e trabalho de investigação, conduzidos por um Júri proposto pela coordenação do projecto ao Conselho Nacional de Especialização Pós-graduada em Ciências Médicas (CNEPGCM), aprovado e nomeado pelo Ministro da Saúde.

\subsubsection{Progressão acelerada do número de internos em} formação e dos dermatologistas formados localmente

A Fig. 3 mostra a evolução do número total de internos em formação a nível nacional de 2012 a 2017. Em 2012, havia sete internos no HAB e a partir de 2013, 14, em 2015, 31 e em 2017, 26 em todos os serviços de formação.

A Fig. 4 mostra o número total de Dermatologistas formados entre 2013 e 2017 dentro e fora do País. Nota-se um aumento de nove especialistas em 2017 ou seja de $62 \%$ relativamente a 2013. Mais de $80 \%$ dos dermatologistas em formação e formados são mulheres. 


\section{Artigo Original}

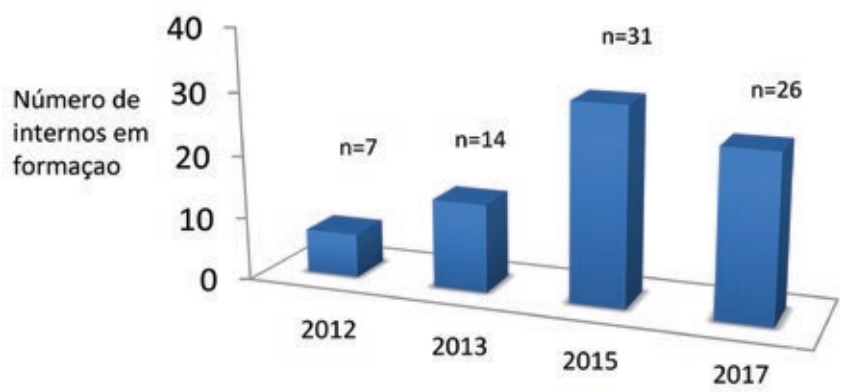

Figura 3 - Formação dos Internos de Dermatologia (2012-2017).

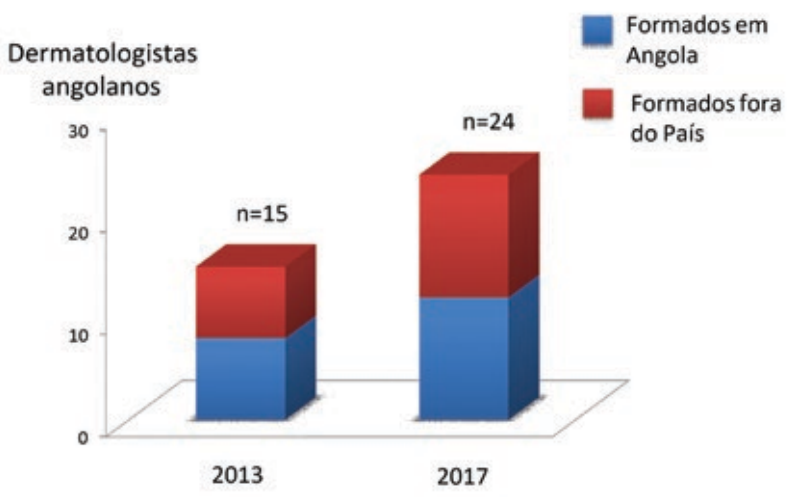

Figura 4 - Dermatologistas Angolanos formados.

A Fig. 5 mostra a curva cumulativa de formação local dos Dermatologistas, passando de oito em 2013 a uma previsão de 23 em 2019.

Dois especialistas beneficiaram duma formação de seis meses em alergologia e cirurgia dermatológica na Clínica de Dermatologia do Centro Hospitalar Universitário de Coimbra.

Alguns internos seniores e tutores nacionais beneficiaram de um apoio financeiro do Projecto para participar em Congressos em Portugal e no Brasil.

Por ocasião do Congresso Anual da Ordem dos Médicos, o CADV oferece desde 2010 cursos anuais de formação contínua em Dermatologia, destinados a internos, médicos de família e outros especialistas angolanos interessados.

\subsection{Investigação}

A partir de 2011, as actividades de pesquisa durante o internato passaram a ser encorajadas e valorizadas no contexto do Projecto. Isso resultou num aumento quantitativo e qualitativo dos trabalhos de pesquisa, no domínio clínico, epidemiológico e operacional, com foco principal no estudo das patologias locais, factor chave na melhoria do acesso, pertinência, qualidade dos serviços e cuidados oferecidos. Entre 2011 e 2017, os internos de Dermatologia

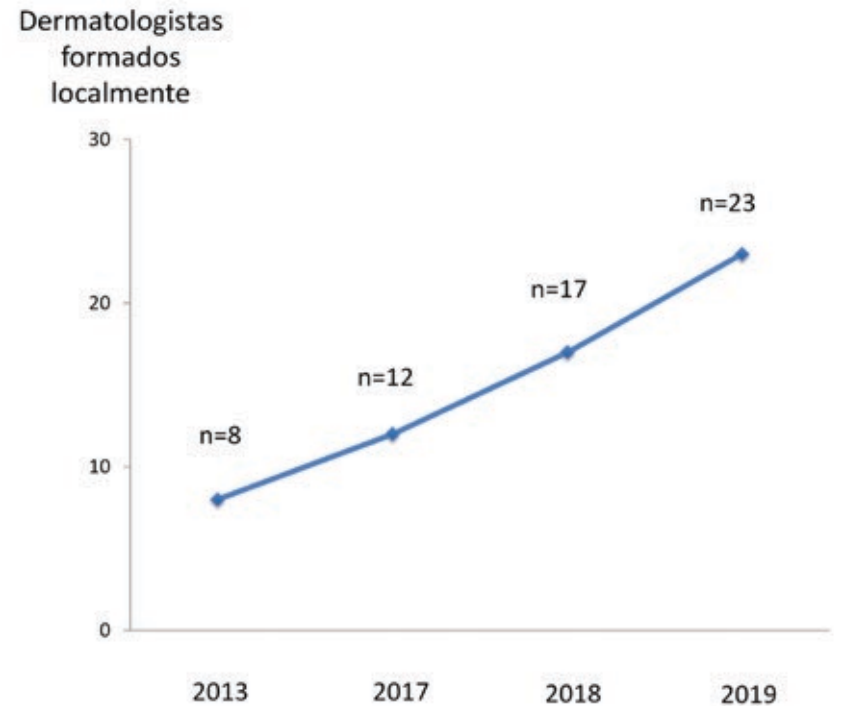

Figura 5 - Evolução da formação Local de Dermatologistas.

apresentaram 21 comunicações orais ou posters em Jornadas Médicas nos Centros Regionais e em Luanda; entre 2016 e o início de 2018 , nove monografias foram defendidas por internos candidatos ao exame de fim do curso de especialização

Por outro lado, o primeiro Simpósio Nacional de Dermatologia, organizado em Luanda em Abril de 2015, mobilizou uma participação nacional e internacional total de cerca de 300 profissionais, incluindo Dermatologistas, internos de especialidade, médicos e técnicos de outras disciplinas. Foram apresentados por quadros nacionais 13 Posters e sete Comunicações orais científicas sobre patologias locais prioritárias, além das 13 conferências apresentadas por especialistas de Portugal, Brasil, Moçambique, China e Angola ${ }^{20}$.

\section{DISCUSSÃO}

O diagnóstico de situação, a definição de objectivos específicos e a estratégia integrada associando assistência clínica, formação médica e pesquisa, criaram condições favoráveis ao desenvolvimento da disciplina. Muitos esforços e paciência foram necessários para modernizar a visão e a abordagem institucional duma especialidade considerada como não prioritária, sem prejuízo da contribuição dada para responder a desafios mais sistémicos tais como a formação, retenção e fixação de especialistas fora da cidade capital, e a substituição gradual da cooperação estrangeira (Congresso da Ordem dos Médicos de Angola, 2018).

Os novos serviços clínicos não cresceram a igual passo. Apesar de terem beneficiado de tutores, espaços, equipamentos e apoio técnico central, os serviços dos Hospitais de Cabinda, Malange e Benguela não conseguiram, até 2015, evoluir com satisfação e apresentar os resultados esperados.

Salvo o HAB, os outros Hospitais de Luanda, em particular o Hospital Josina Machel (HJM), acabaram por não 


\section{Artigo Original}

demonstrar idoneidade para formar internos em Dermatologia.

Persistem algumas carências importantes, tais como uma unidade de histopatologia que possa processar e analisar rapidamente e com qualidade as biopsias cutâneas a nível nacional. Este problema afecta igualmente outros países do continente. Apesar de progressos notáveis, existe ainda em África, um défice significativo na formação de dermatopatologistas e na disponibilidade de laboratórios de histopatologia com qualidade. ${ }^{8}$

Verificou-se que para garantir a reposição regular de alguns produtos específicos prioritários (alergénios, azoto líquido, testes rápidos e reagentes laboratoriais), a supervisão regular a nível regional, o acesso a internet e, entre outros, a efectivação do estágio de seis meses em serviços fora do país para os internos que já terminaram ou vão terminar a especialização, o Projecto deve dispor de meios financeiros reservados.

A nível da formação, a motivação, a competência e o empenho da equipa de formadores exercem um papel determinante na atração, qualidade da formação e retenção dos internos. Alguns supervisores e tutores não assumem contudo de forma consistente a sua função de liderança e reforço institucional, facto atribuído a ausência de compensação profissional e contratual da parte do MINSA, ou seja a definição e aplicação de um regime de dedicação exclusiva ao Hospital e a formação dos internos.

A semelhança do que acontece no ensino pré-graduado, ${ }^{12}$ foi constatado que as Inovações metodológicas e a harmonização do currículo de formação dos internos, métodos de aprendizagem, supervisão, monitoria e avaliação são elementos essenciais para garantirem, a nível nacional, a qualidade dos especialistas formados.

Tendo em consideração a escassez de tutores, o apoio didáctico prestado por internos seniores a internos juniores, merece ser encorajado e sistematizado de forma controlada, como já observado e recomendado em outros países. ${ }^{22-24}$

Entretanto, a cultura geral clínica e científica adquirida a nível pré-graduado ainda não atinge em Angola o nível preconizado. ${ }^{12} \mathrm{O}$ investimento pessoal dos internos durante a sua formação pós-graduada deve portanto compensar determinadas insuficiências, na óptica que a formação não se limita a mera difusão de conhecimentos e aos ensinamentos dos tutores. A elevada carga horária obrigatória dos internos nos Bancos de Urgência de Medicina, contribui contudo a limitar o tempo de estudo e aprendizagem pessoal.

A semelhança de outros países, ${ }^{24-26}$ a avaliação dos currículos anuais dos internos nos informa que a aprendizagem e a prática dos actos técnicos (cirurgia, alergologia, dermatoscopia, dermatologia cosmética), bem como as competências laboratoriais (histopatologia, micologia, bacteriologia e parasitologia) estão ainda abaixo dos níveis preconizados. Partindo do princípio que a disciplina é principalmente ambulatória, o volume de pacientes vistos semanalmente por cada interno na consulta externa deve ainda aumentar para que cada um possa adquirir a competência desejada.
A apreciação que têm os internos sobre os formadores e o programa de formação em geral não deve ser esquecida. Está em curso a elaboração de um formulário de avaliação do grau de satisfação e das sugestões dos internos em relação ao programa e as condições de formação, uma mais-valia demonstrada em diversos países. ${ }^{6,26}$

Verificou-se que a troca de experiência e a colaboração entre o MINSA, as Faculdades de Medicina proporciona benefícios mútuos, na busca de soluções a desafios comuns a nível pré e pós-graduado, como a introdução de novas abordagens curriculares, novos métodos de aprendizagem, e promoção da investigação. ${ }^{12}$

Apesar dos progressos registados, a investigação em dermatologia regista várias fraquezas, nomeadamente nas habilidades informáticas e metodológicas dos internos, factores limitantes no tipo e qualidade dos trabalhos apresentados. As comunicações e publicações produzidas acabam por ser, na sua grande maioria, clínicas, descritivas e retrospectivas, reflectindo uma fase ainda incipiente na prática da investigação. ${ }^{27}$

Recomenda-se dedicar mais atenção a formação metodológica dos internos e tutores na área da investigação, bem como um aumento dos meios financeiros destinados a pesquisa, actualmente quase inexistentes. Estudos indicam que a interacção e o envolvimento precoce com a produção e a crítica científica resultam em maior quantidade e melhor qualidade na produção futura. ${ }^{27}$

\section{CONCLUSÕES E RECOMENDAÇÕES}

A dermatologia em Angola ainda encontra-se numa fase precoce do seu desenvolvimento. Porém, a partir de 2010, e apesar de inúmeros desafios, foram registados avanços positivos no desenvolvimento da disciplina e na formação de dermatologistas.

Fazemos nossa a observação do Prof. Américo Figueiredo, ${ }^{28}$ "Muitos jovens de qualidade entraram e estão a entrar em Dermatologia escolhidos de entre os melhores, e com vontade e necessidade de dar um novo impulso a Dermatologia. Necessitamos de os incentivar, de lhes indicar os caminhos possíveis e de lhes preparar as ferramentas e a oportunidade de poderem ter sucesso a bem da Dermatologia, da Medicina e, em última análise, dos seus futuros doentes".

A sustentabilidade das acções em curso requer do nível institucional, um compromisso político efectivo, recursos humanos empenhados e recursos financeiros adequados para garantir a qualidade da formação bem como as condições de trabalho, a motivação, o empenho e a adesão profissional dos tutores e internos.

O diagnóstico e tratamento precoces das patologias genéticas, tumorais e inflamatórias, diagnosticadas com maior frequência em Angola, exigem a aquisição de novas competências e tecnologias, sendo prioritária a criação de uma unidade de histopatologia cutânea e treino em dermatopatologia.

Deve ser reforçada a investigação em domínios prioritários no contexto nacional, bem como a formação dos 


\section{Artigo Original}

internos na metodologia científica, incluindo a elaboração e redacção obrigatória de uma monografia ou artigo científico, a ser concluído até o final da formação.

A experiência do Projecto de Dermatologia poderá incentivar estudos adicionais sobre a estrutura e o desempenho de outros programas de pós-graduação em Angola, incluindo a avaliação do seu impacto na formação, distribuição geográfica e retenção de especialistas fora da cidade capital.

As parcerias internas e externas são mecanismos essenciais para garantir a qualidade e a sustentabilidade dos ganhos, incluindo a formação de quadros subespecializados.

Propõe-se a criação em Luanda de um Centro de Formação em Dermatologia, para dinamizar o desenvolvimento da disciplina, incluindo a formação de quadros, troca de experiência e investigação em Angola e nos outros países lusófonos da Região.

\section{Agradecimentos}

Os meus agradecimentos vão a todos os Dermatologistas do Colégio Angolano de Dermatologia e Venereologia que participam no Projecto, em particular ao Dr. Pascoal O. Alberto e Dr. Juliano I. Vicumba; agradecemos o Prof. Miguel Bettencourt, ex- Decano da Faculdade de Medicina da Universidade Agostinho Neto em Luanda, por me ter autorizado a realizar as visitas as Províncias, bem como aos Prof. Albano VL Ferreira e Cristóvão Simões, respetivamente Reitores das Universidades Katyavala Bwila em Benguela e José Ed. dos Santos no Huambo, pela revisão do manuscrito.

Conflitos de interesse: Os autores declaram não possuir conflitos de interesse.

Suporte financeiro: O presente trabalho não foi suportado por nenhum subsídio ou bolsa.

Confidencialidade dos dados: Os autores declaram ter seguido os protocolos do seu centro de trabalho acerca da publicação dos dados de doentes.

Protecção de pessoas e animais: Os autores declaram que os procedimentos seguidos estavam de acordo com os regulamentos estabelecidos pelos responsáveis da Comissão de Investigação Clínica e Ética e de acordo com a Declaração de Helsínquia da Associação Médica Mundial

Conflicts of interest: The authors have no conflicts of interest to declare.

Financing Support: This work has not received any contribution, grant or scholarship.

Confidentiality of data: The authors declare that they have followed the protocols of their work center on the publication of data from patients.

Protection of human and animal subjects: The authors declare that the procedures followed were in accordance with the regulations of the relevant clinical research ethics committee and with those of the Code of Ethics of the World Medical Association (Declaration of Helsinki).

\section{REFERÊNCIAS}

1. Gomes TM, Miranda Soares de Moura A T, Cavalcanti de Aguiar A. Dermatologia na Atenção Primária: um Desafio para a Formação e Prática Médica. Rev Brasil Edu Méd. 2012; 36: 125-8.

2. Pitché $P$, Tchangai-Walla K. La dermatologie en Afrique Noire, quelles perspectives pour le 21 e siècle? Nouv Dermatol. 2000; 19: 44-8.

3. Pereira A. O. Contribuição ao estudo do perfil nosológico das consultas ambulatórias nos centros de saúde em Luanda. Luanda: Faculdade de Medicina da Universidade Agostinho Neto; 2009.

4. Fortes F. Doenças Tropicais Negligenciadas em Angola e a Dermatologia. Comunicação no $1^{\circ}$ simpósio nacional de Dermatologia, Luanda, 2015.

5. Hay R, Estrada R, Grossmann H. Managing skin disease in resource-poor environments - the role of community-oriented training and control programs. Int J Dermatol. 2012; 50: 558-63.

6. Nkabinde TC, Ross A, Reid S, Nkwanyana NM. Internship training adequately prepares South African medical graduates for community service - with exceptions. S Afr Med J. 2013; 103:930-4.

7. Lowell BA, Froehlich CW, Federman DG, Kirsner RS. Dermatology in primary care: Prevalence and patient disposition. J Am Acad Dermatol. 2001; 45:250-5.

8. Beltraminelli H. 2018 Africa. Where do we stand in Dermatopathology training? Oral communication, 4th African Dermatology Conference, RDTC Moshi, 2018.

9. Völker K. Tropenmedizinisch/tropendermatologische Ausbildung in Tansania und Ghana. Ein Erfahrungsbericht. Hautarzt. 2015; 66:311-319

10. Regional Dermartology Training Centre [consultado 04.01.2018] Disponível em:http://rdtc.go.tz/index.html

11. XLIV Congresso Brasileiro de Dermatologia- Simpósio sobre Pós-graduação em Dermatologia. An Bras Dermatol. 1990; 65

12. Ferreira A V, Fresta M, Simões CF, Do Rosário SM. Desafios da educação médica e da investigação em saúde no contexto de Angola. Rev Edu Méd Brasil. 2004; 38: 133-41.

13. Ordem dos Médicos de Angola. Regulamento Geral do Colégio De Dermatologia e Venereologia de Angola CADV. Luanda: OMA; 2010.

14. Projecto de Desenvolvimento da Dermatologia a Nível Nacional. Luanda: MINSA; 2010.

15. Voumard A.L. Estudo comparativo da formação pré e pós-graduada em Dermatologia no Pequim First University Hospital e na FMUAN. 2004.

16. Decreto Presidencial No 17/04 de 31 de Maio sobre o internato complementar médico. Diário da República, Luanda 2004.

17. Decreto Presidencial No $260 / 10$ de 19 de Novembro sobre o Regime Jurídico da Gestão Hospitalar. Diário da República, Luanda 2010.

18. Decreto Presidencial No $271 / 11$ de 26 de Outubro 


\section{Artigo Original}

sobre o internato complementar médico. Diário da República, Luanda 2011.

19. Proposta de Regulamento geral do Interno de Especialidade no Hospital Américo Boavida, Conselho Directivo do HAB, Deliberação No 10/CD/HAB/2015.

20. Proposta de critérios para o reconhecimento da idoneidade dos hospitais e serviços para realização de internatos de especialidades. Luanda: MINSA; 2017.

21. Projecto de Dermatologia: Relatório do $1^{\circ}$ Fórum sobre o Regulamento e Organização dos Serviços de formação em Dermato-Venereologia em Angola. Luanda: MINSA; 2013.

22. Projecto de Dermatologia: Relatório sobre o Acto Central de Abertura Oficial dos Serviços Regionais de Dermatologia. Luanda: MINSA; 2013.

23. Projecto de Dermatologia: Programa do $1^{\circ}$ Simpósio Nacional de Dermatologia e Venereologia, Luanda, Abril 2015.
24. Burgin S, Homayounfar G, Newman LR, Sullivan A. Instruction in teaching and teaching opportunities for residents in US dermatology programs: results of a national survey. J Am Acad Dermatol. 2017; 76: 703-6.

25. Plee J, Barbe C, Richard M-A, Dréno B, Bernard P. Etat des lieux de la formation des internes en DES de Dermato-Vénéréologie en France (2005-2010). Ann Dermatol Vénéréol. 2013; 140: 259-65.

26. AlGhamadi KM. Current status of Dermatology residency training in Saudi Arabia: trainee's perspective. East Mediterr Health J. 2008; 14: 1185-91.

27. Alencar Marques S, Amante Miot H, Fernandes Abbade LP. Produção científica publicada nos Anais Brasileiros de Dermatologia (2003-2007). An Bras Dermatol. 2008; 83:555-600.

28. Figueiredo A. Dermatologia do seculo XXI: que desafios? Que oportunidades? Comunicação no $1^{\circ}$ simpósio nacional de Dermatologia, Luanda, 2015. 